\title{
Vorwort des Autors
}

Das vorliegende Buch verfolgt einen pragmatischen Ansatz. Es soll System-Entwickler beim Entwurf, der Modellierung und der Realisierung von Erweiterungen relationaler Datenbanken um deduktive Fähigkeiten unterstützen. Diese Erweiterungen dienen der Realisierung von leistungsfähigen Informationssystemen.

Die Motivation zur Erstellung dieser Monographie resultiert aus der Uberzeugung, daß die praktische Relevanz von Konzepten, die aus der Interdisziplinarität der Bereiche Datenbanken und Künstliche Intelligenz $(\mathrm{DB} / \mathrm{KI})$ entstanden sind, einen signifikanten Beitrag zur Realisierung von Anwendungen auf existierenden relationalen Datenbeständen liefern kann.

Jedoch müssen die hier aufgezeigten Konzepte zumindest transparent sein bezüglich der Anwendung sowie integriert im Rahmen der existierenden Arbeitsumgebung, damit bei ihrem Einsatz ein Durchbruch erzielt werden kann. Das vorliegende Buch unternimmt diesen Versuch. Bei der Erstellung dieser Monographie wurde von der Annahme ausgegangen, daß der Leser - im Vergleich - über mehr Wissen zu Datenbanken verfügt als zu den Technologien der Künstlichen Intelligenz.

Deshalb werden die Grundlagen im Bereich des logischen Programmierens formal beschrieben mit dem Ziel, den Leser in diese Begriffswelt einzuführen. Das spezifizierte Konzept (hier KANON genannt) soll als Basis zur Realisierung unterschiedlicher konzeptueller Schemata dienen. Die dafür notwendige Voraussetzung ist durch die ausführliche Spezifikation der zugrundeliegenden Metaschemastruktur gegeben.

Inwieweit die Metastrategien, die Constraint-Mechanismen und die Editoren wie Regel-Editor, Metaregel-Editor und Constraint Enforcement-Editor als Werkzeuge zur Vereinfachung der Interaktion einer mit dem KANON-Ansatz realisierten Anwendung dienen sollten, ist primär aus der Benutzeranforderung abzuleiten.

Da die Nutzung von existierenden relationalen Datenbeständen von besonderer Bedeutung ist, werden die prinzipiellen Kopplungsansätze beschrieben, die den Zugang zu existierenden Daten ermöglichen. Weiterhin sind neue Kopplungsstrategien und die dazugehörigen Prädikate zum Wissens- und Datentransfer ausführlich erläutert. 
Die Tragfähigkeit des hier vorgestellten Konzepts wurde durch eine prototypische Implementierung in PROLOG mit einer relationalen Datenbank getestet. Dies hat natürlich einen Einfluß auf die syntaktische Darstellung der Inhalte des Metaschemas zur Folge; die Implementierung dieses Metaschemas in dieser Monographie könnte in einer beliebigen Programmiersprache wie auch im Rahmen einer Anwendung auf der Basis einer relationalen oder objektorientierten Datenbank stattfinden.

Das hier definierte Konzept soll als Spezifikationen für mögliche Realisierungen dienen. Natürlich können und sollen nur die Teile des vorgestellten Modells ausgesucht werden, die zur Realisierung der jeweiligen Anwendung auch tatsächlich benötigt werden.

Mein Dank gilt allen, die mich bei der Erstellung dieses Buches unterstützt haben! 